\title{
The Design of Shared Aperture Antennas Consisting of Differently Sized Elements
}

\author{
Cristian I. Coman, Student Member, IEEE, Ioan E. Lager, Member, IEEE, and Leo P. Ligthart, Fellow, IEEE
}

\begin{abstract}
A consistent strategy for the design of finite array antennas consisting of differently sized radiating elements is discussed. In view of increasing the total bandwidth of the antenna system, while maintaining a low mutual coupling between the elementary radiators, sparse subarrays, operating at adjacent frequency ranges, are interleaved on a common (shared) aperture. The sparse architectures are designed using a combinatorial method that ensures an acceptable behavior in the side-lobes region in conjunction with a narrow beamwidth. The effect of the mutual coupling between identical and differently sized elements is accurately evaluated and is accounted for in predicting the performance of each individual radiator. The concept is illustrated by designing a shared aperture antenna consisting of two interwoven subarrays that jointly cover a fractional bandwidth of $14 \%$ in the $\mathrm{X}$-band. Cavity-backed, stacked-patches antennas with coaxial feeding are used as elementary radiators.
\end{abstract}

Index Terms-Mode-matching methods, shared aperture, sparse array antennas.

\section{INTRODUCTION}

$\mathbf{M}$ ULTIFUNCTIONALITY of the antenna systems is a key issue in the case of mobile platforms performing simultaneously multiple tasks, such as communication, remote sensing, electronic warfare, etc. Traditionally, there is a twofold approach to ensuring the multifunctionality of these systems [1, Sec. 2.7]:

- time sharing (time multiplexing) — the antenna resources are employed sequentially by different systems at distinct time slots;

- aperture sharing (aperture segmentation) - separate radiators are employed for concurrently serving each required functionality; since, more often than not, the space for deploying antennas is limited, the relevant radiators have to be closely packed.

While the former approach is, in principle, compatible with any type of antenna, the latter implies, necessarily, the use of a collection of elementary radiators that are integrated in an antenna array. In it, each functionality is associated with a specific subarray.

Upon focusing on the aperture sharing approach, it is noted that it can be implemented by placing on the relevant aperture either identical or different radiators. The former choice [2], [3] allows a facile (possibly dynamic) reconfiguration of the aperture.

Manuscript received January 14, 2005; revised May 12, 2005.

The authors are with the International Research Centre for Telecommunications-Transmission and Radar (IRCTR), Delft University of Technology, 2600 GA Delft, The Netherlands (e-mail: c.coman@irctr.tudelft.nl; i.lager@ its.tudelft.nl; 1.p.ligthart@irctr.tudelft.nl).

Digital Object Identifier 10.1109/TAP.2005.863382
However, the various tasks to be accomplished are often associated with spectra that are spread over a wide frequency range and, consequently, the antenna elements need to be of the widerange variety. Apart from the fact that these type of antennas have, intrinsically, a large electric size (that becomes a critical issue when beam-scanning is aimed at) this approach also yields a considerable interference between the implemented functions as a result of the out-of-band mutual coupling. When using different radiators, they can be tuned for each individual application, thus improving the isolation between the different systems. A straightforward modality for achieving an aperture sharing is by deploying next to each other a number of compact subarrays [1, p. 206]. However, each subarray is confined to a small area, thus providing but limited angular resolution. To sidestep this drawback, the radiators pertaining to the various subarrays may be distributed over the entire available area, in an interleaved manner, for example by deploying the elementary antennas on interlaced, uniform grids [4]-[6]. While this option is conceptually simple, it results in severe limitations as far as scanning range, polarization, and the ratio between the frequency bands pertaining to the subarrays are concerned.

Most of the deleterious aspects mentioned above can be circumvented by calling upon the use of interwoven, sparse arrays. To substantiate this, it is noted that

- by applying an adequate thinning technique, in conjunction with an interweaving procedure that avoids the overlapping of the elements, the grating lobes (that would occur in the case of widely spaced, uniform architectures) are suppressed;

- the side-lobes level can be controlled without resorting to amplitude tapering (that would be needed in uniform arrays); and

- the large interelement distances results into a reduced in-band mutual coupling.

The present contribution advocates a multifunctional antenna architecture based on the shared aperture, sparse array antenna approach [7]. For implementing this concept, two aspects are of paramount importance: the design of the individual radiators functioning in nonperiodic, finite array environments, and the evaluation of the radiation properties of the densely populated shared apertures, while also accounting for the effect of the mutual coupling. To this end, a novel, consistent design strategy, having as main purpose to ensure that all radiators in the array configuration do radiate adequately, is proposed. The numerical approach underlying this strategy relies on the decomposition of the electromagnetic problem at hand into an external and an internal problem, the former addressing the radiation 


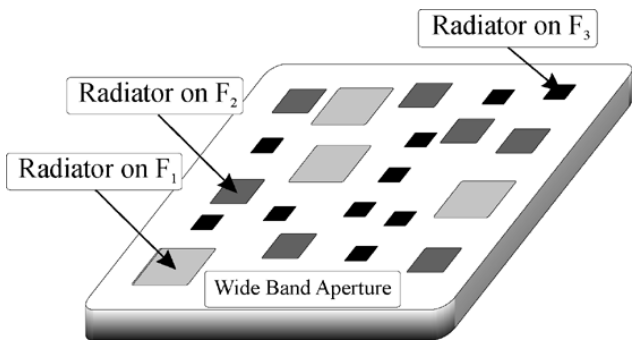

Fig. 1. Illustrative for the shared aperture antenna concept.

phenomena and the latter the matching between the elementary feeding structures (down to the coaxial cables) and the free-space.

The account now proceeds with a detailed description of the adopted design philosophy. As a first step toward obtaining the antenna system that is aimed at, a deterministic thinning method, corroborated with a suitably chosen interweaving strategy, is applied for the placement of the individual radiators. Subsequently, the solution of the external problem is discussed by evaluating the interelement mutual coupling, as well. The result of this step is a Generalized Admittance Matrix (GAM) that models the radiation from the complete structure, that provides, in turn, the boundary conditions for the internal problem concerning each elementary antenna. The proposed algorithm is illustrated by means of designing a shared aperture antenna consisting of two interwoven subarrays that use as elementary radiators cavity-backed, stacked-patches antennas with coaxial feeding.

\section{DESIGN STRATEGY}

The antenna architecture promoted by the present work is schematically illustrated in Fig. 1. The rationale of this concept relies on the fact that, by eliminating a (sizeable) number of array elements as a result of applying a thinning technique, a lot of space becomes available on the aperture. This space can then be occupied by radiators belonging to another subarray, providing the overlapping of elements is prevented. Note that by using this strategy for subarrays with distinctive, but contiguous operational bandwidths, an (ultra-) wide-band antenna system can be synthesized.

The realization of this concept assumes addressing a number of important aspects:

- the choice for a type of primary radiator;

- the choice for a suitable thinning technique;

- the design of an interleaving procedure that is consistent with the previously chosen thinning technique;

- the rigorous analysis of the internal and external problems, as defined in the concluding part of Section I.

These aspects are hereafter examined individually.

\section{A. The Choice for the Type of Primary Radiator}

Currently, there is a manifest trend toward developing lowprofile and low-cost antenna systems. Frequently, a dual polarization requirement is formulated, as well. A popular answer to this set of demands is provided by the class of (stacked) microstrip patch antennas [8]-[11]. These are moderate to wide bandwidth radiators, covering fractional frequency ranges from 5 to $75 \%$. When patch antennas are employed in array environments, the surface waves that are generated (the more so in the case of stacked-patches antennas) have a detrimental effect of the antenna's efficiency, especially at large scanning angles [12]. The most adequate solution to the suppression of the surface waves is to place the radiating patch(es) in a metallic cavity $[13]-[15]$. Note that this solution also contributes to the reduction of the mutual coupling between elements. For feeding such radiators, either protruding probes [8], [14], [15] or an aperture coupling [10] have been proposed. While the latter option is generally considered to ensure a wider operational bandwidth, the former provides a better isolation between the channels and a more direct access to individual elements, that is essential for measurement purposes (a highly relevant aspect during the system development phase).

By accounting for all these features, a cavity-backed, stackedpatches antenna, fed by means of a coaxial probe is selected as preferred elementary radiator.

\section{B. The Choice for the Thinning Technique}

Presently, there is a vast number of thinning techniques that are suitable for designing sparse arrays (see the overview on this topic in [16]). On an overall, these methods can be categorized as either deterministic or stochastic. The latter allow a larger flexibility in the choice for the parameters that are subject to optimization and for the constraints. Nevertheless, they are characterized by slow convergence that yields very long computation times. Consequently they are considered unsuitable for the design of sparse arrays containing many elements, the kind of which are at the focus of the present work. When turning to deterministic techniques, the combinatorial approach, most notably the difference sets method [17], [18], are considered as the best candidates for designing the subarrays to be interwoven.

By definition, a $(V, K, \Lambda)$ difference set is a set of $K$ unique integers $D=\left\{d_{0}, d_{1}, \ldots, d_{K-1}\right\}$, with $0 \leq d_{i} \leq(V-1)$, such that for any integer $1 \leq \alpha \leq(V-1)$

$$
\left(d_{i}-d_{j}\right)(\bmod V)=\alpha, \text { for } i \neq j
$$

has exactly $\Lambda$ solution pairs $\left\{d_{i}, d_{j}\right\}$ from the set $D$, with "mod" standing for the "modulo" operation. Note that the set $D^{*}=$ $[0, V-1] \backslash D$ (referred to as the complementary of $D$ ) is also a difference set with parameters $(V, V-K, V-2 K+\Lambda)$.

From a difference set $D$ having the parameters $(V, K, \Lambda)$, one can construct a sequence of ones and zeros

$$
A_{D}(i)= \begin{cases}1 & \text { if } i \in D \\ 0 & \text { if } i \notin D\end{cases}
$$

with $i=0,1, \ldots, V-1$. The $A_{D}$ sequence exhibits some remarkable properties. In [18] it was indicated that the autocorrelation function of an infinite vector obtained by periodically repeating $A_{D}$ is a two-valued function. It was also noted that the discrete Fourier transform of the sequence $A_{D}$, taken on $V$ points, has only one maximum at a level that equals $K$, whereas all the other spectral components have an identical, low value (related to $\Lambda$ ).

By placing the elements in a linear thinned array at the positions of the ones in $A_{D}$, the antenna radiation pattern preserves 
the beamwidth of the full array (consisting of $V$ uniformly distributed radiators) whereas the side lobe level has approximately a constant value of $1 /(2 K)$, relative to the main beam [18].

A planar thinned array of dimensions $V_{x}$ and $V_{y}$ can be obtained from the difference set $D$ with parameters $(V, K, \Lambda)$ by defining the matrix of one and zeros

$$
M_{D}[i(\bmod V x), i(\bmod V y)]= \begin{cases}1 & \text { if } i \in D \\ 0 & \text { if } i \notin D\end{cases}
$$

with $i=0,1, \ldots, V-1$ and $V_{x} V_{y}=V$. Using one set from the elements of matrix $M_{D}$ (ones or zeros) to deploy the radiators in the sparse configuration, the power pattern of the thinned array has a beamwidth similar to the one of the full planar array, whereas the side-lobe level has approximately a constant, low value.

\section{The Interleaving Procedure}

Contrary to the thinning techniques, this topic is seldom encountered in the literature. The main goal of this design step is to preserve the sparsity properties of the subarrays (especially in the sense of avoiding the generation of grating lobes), while preventing the overlapping of elementary radiators. A viable solution to this design problem was described in [19]. That approach is at the origin of the strategy applied in the present contribution, as well.

\section{The Analysis of the Internal and External Problems}

The solution of the electromagnetic problems concerning the analysis of the cavity-backed patch antennas can be obtained by either resorting to a three-dimensional grid (as in the case of the finite-differences and finite-element methods [13], [20]) or by applying an integral-equations approach (as in the case of the mode-matching method [21]-[23]). For efficiency considerations, the design strategy discussed in this work employs a mode-matching technique, in which the modes on the noncanonical waveguide sections are evaluated by means of the boundary-integral, resonant modes expansion (BI-RME) method [23].

The evaluation of the radiation parameters is traditionally carried out by either isolating the elementary radiators and analyzing them outside the array environment or by accounting for an infinitely extended (periodic) array. The former approach has as main drawback the fact that the radiation properties of the individual elements are significantly affected when placing them in the array environment. An example in this sense is provided in [24], [25] where including the excellent type of radiator described in [10] in a $5 \times 5$ array results in a massive deterioration of the matching properties in the case of the elements located at the center of the array. The infinitely extended array correctly addresses the radiation properties in array antennas, on the provision that true periodicity of the structure is enforced. The use of this model is, nevertheless, not applicable to the case of the sparse subarrays under investigation since no periodic properties can be asserted to them. The present paper proposes a full-wave analysis of the complete radiating structure, such that the behavior of the elements in the array environment to be consistently taken into account.

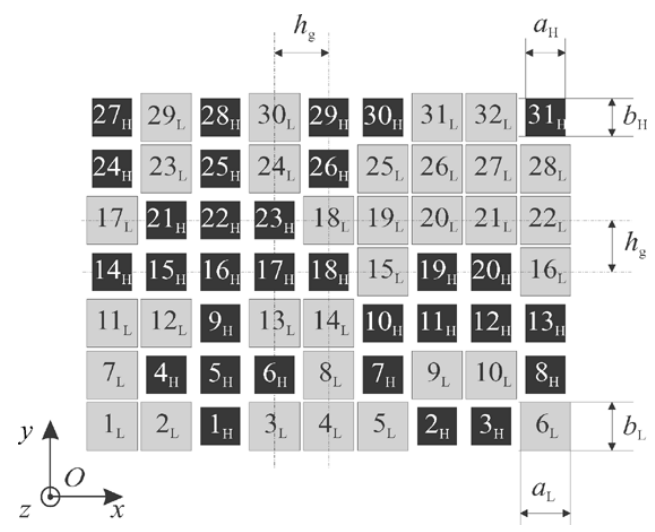

Fig. 2. Distribution of the elementary antennas on the shared aperture. The step of the grid is chosen $h_{\mathrm{g}}=15 \mathrm{~mm}$. The dimensions of the radiators are still to be specified.

\section{IMPLEMENTATION OF THE DESIGN STRATEGY}

The design strategy described in the Section II is demonstrated for the case of a shared aperture antenna consisting of two subarrays operating at adjacent frequency ranges, that must jointly cover a fractional frequency band of at least $10 \%$ within the X-band. The subarrays are hereafter referred to as $\mathrm{SA}_{\mathrm{H}}$ and $S A_{L}$, where the indexes " $H$ " and " $L$ " designate the higher and the lower frequency bandwidths, respectively.

The objective is to obtain a medium-sized array antenna offering scan capabilities in the hemispherical field of view. The antenna must be amenable to manufacturing in a printed, stratified technology, by using commercially available, high-frequency, plated laminates.

\section{A. The Design of the Sparse Array's Architecture}

The first step in the design algorithm is represented by the determination of the array's architecture. Preliminary analyses have shown that it is possible, by using a dielectric substrate with high permittivity (RO54350B, having $\varepsilon_{\mathrm{r}}=3.48$ [26]), to reduce the maximum size of the elements in the $\mathrm{SA}_{\mathrm{L}}$ subarray below the value $\lambda_{\mathrm{X}, \min } / 2$, with $\lambda_{\mathrm{X}, \min }$ denoting the wavelength at the upper limit of the X-band $(10 \mathrm{GHz})$. (The interrelation between frequency and size implies that the elements in the $\mathrm{SA}_{\mathrm{H}}$ subarray are smaller.) Consequently, a common grid, having square cells with edge lengths of $\lambda_{\mathrm{X}, \mathrm{min}} / 2=15 \mathrm{~mm}$, can be employed for deploying all elements. After having selected the grid, the design proceeds by applying the difference sets method. In this respect, the difference set DS $=(63,51,15)$ is used for deploying the elements of the $\mathrm{SA}_{\mathrm{H}}$ subarray, while its complementary provides the positions of the radiators of the other one. This automatically resolves the interleaving problem, since no overlapping is possible anymore. Note that the possibility to use a common grid in conjunction with the difference set method results in a substantial simplification of the placement algorithm when compared to the one discussed in [19].

The result of the application of the above placement procedure is depicted in Fig. 2. In it, the black boxes designate the area allocated to the radiators in the $\mathrm{SA}_{\mathrm{H}}$ subarray and the gray ones the area allocated to the $\mathrm{SA}_{\mathrm{L}}$ subarray. Furthermore, for being able to describe the directions of radiation in the free-space, a Cartesian reference frame, having the origin $\mathcal{O}$ located at the 


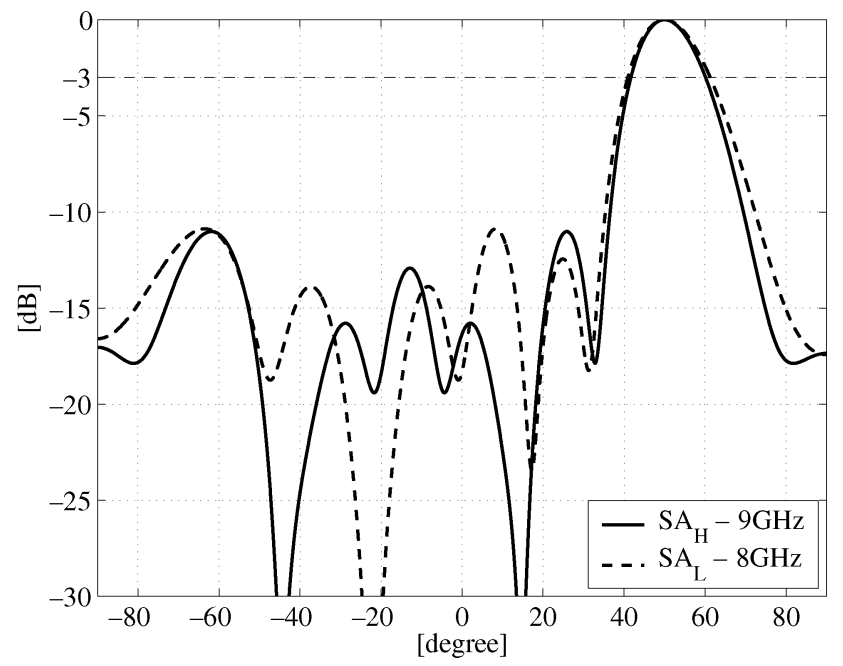

Fig. 3. Array factor in the case of a beam-scanning at $\vartheta=50^{\circ}$ and $\varphi=0^{\circ}$. The solid line corresponds to the $\mathrm{SA}_{\mathrm{H}}$ subarray (at a frequency of $9 \mathrm{GHz}$ ) and the dashed one to the $\mathrm{SA}_{\mathrm{L}}$ subarray (at a frequency of $8 \mathrm{GHz}$ ).

aperture's plane and the orientation specified in the figure, is accounted for. The elevation angle is taken with respect to the $\mathcal{O} z$ axis and is denoted as $\vartheta$ while the azimuth angle, measured in the $x \mathcal{O} y$ plane with respect to the $\mathcal{O} x$ axis, is denoted as $\varphi$.

By examining Fig. 2, it is obvious that the deployment strategy uses the available real estate in a highly efficient manner, since the nonradiating part of the aperture is insignificant. The radiation properties of the designed shared aperture antenna are illustrated by plotting the array factor for the case when the main beam was scanned to $\vartheta=50^{\circ}$ and $\varphi=0^{\circ}$ (see Fig. 3). Note that this is just a preliminary test, carried out for ideal (isotropic) radiators and by neglecting the mutual coupling. The $-3 \mathrm{~dB}$ beamwidth is narrower than $20^{\circ}$ in the $x \mathcal{O} z$ plane. At the same time, it can be observed that the side-lobes level is below $-10 \mathrm{~dB}$.

\section{B. Characterization of the Primary Radiators}

After defining the grid for deploying the elements, the next step is the design of the elementary radiators. Initially, the radiators in each subarray are taken to be identical. Let $R_{H}$ and $R_{L}$ be the elements of the subarrays operating at the high and the low frequencies, respectively. The topology of the generic radiator (that is representative for both $R_{H}$ and $R_{L}$ ) is depicted in Fig. 4. It consists of two stacked circular patches, the lower one being fed by means of a protruding pin. The two patches are etched on commercially available microstrip laminate (RO54350B, having $\varepsilon_{\mathrm{r}}=3.48$ ) and are stacked together inside a metallic cavity. Both patches are centered with respect to the cavity. The thicknesses of the high-frequency laminates are identical in the case of both types of radiators, namely $h_{1}=0.338 \mathrm{~mm}, h_{2}=1.524 \mathrm{~mm}$ and $h_{3}=h_{1}$. The thickness of all metallic layers amounts to $h_{\mathrm{m}}=0.07 \mathrm{~mm}$. Note that all dimensions were chosen in compliance with the data specifications of the employed microwave material. Furthermore, by choosing laminates of identical thicknesses for both $R_{H}$ and $\mathrm{R}_{\mathrm{L}}$, the conditions are prepared for manufacturing the aperture antenna in a printed, stratified technology. The dimensions of the coaxial cable replicate those of a subminiature, type A (SMA) connector $\left(r_{\text {in }}=0.64 \mathrm{~mm}\right.$ and $r_{\text {out }}=2.05 \mathrm{~mm}$ ), the
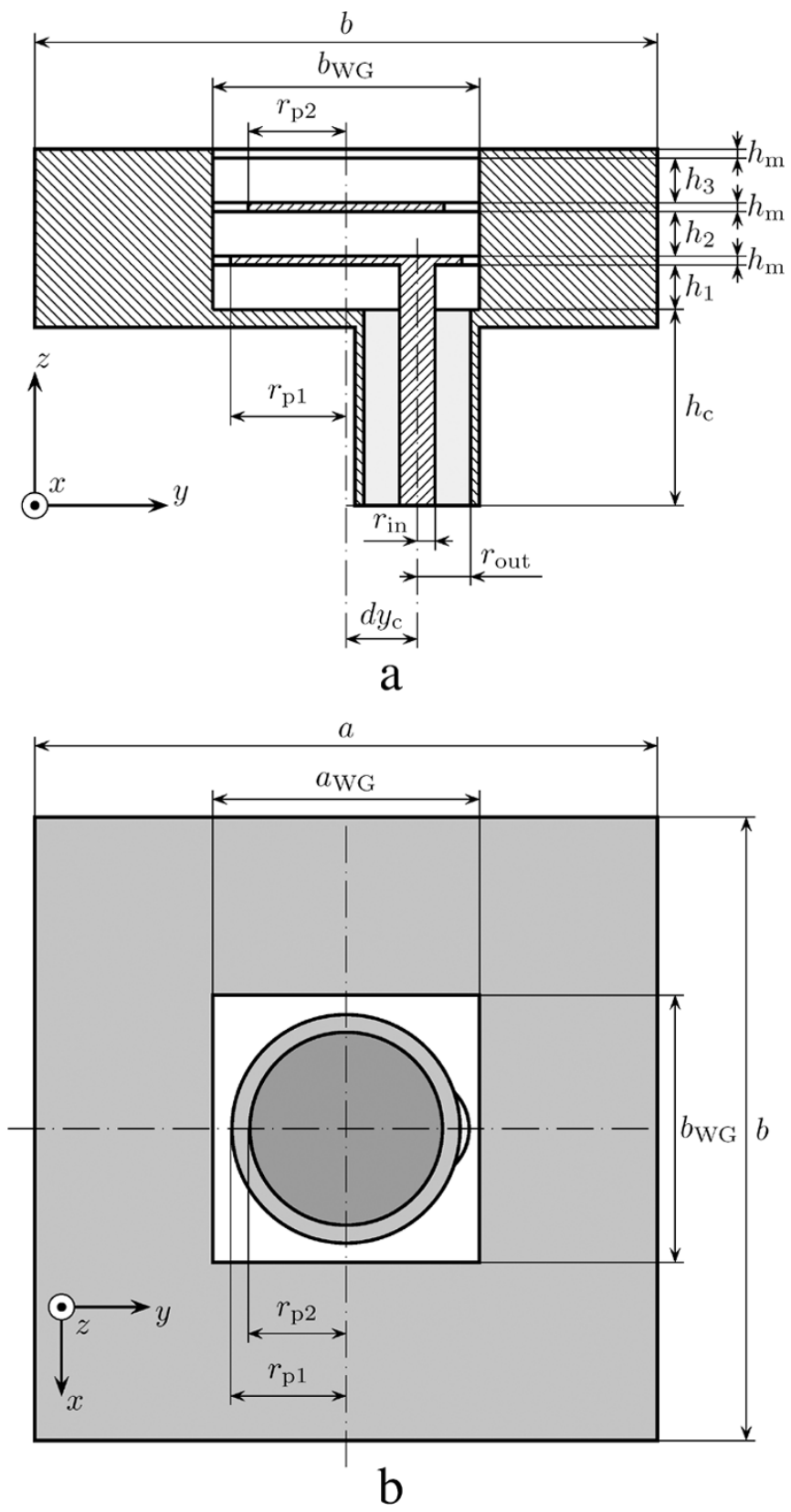

Fig. 4. Configuration concerning the double patch antenna. (a) Axial cross section. (b) Top view.

relative dielectric permittivity inside it being $\varepsilon_{\mathrm{r}, \mathrm{c}}=1.951$. The free design parameters are the dimensions of the cavity ( $a$ and $b$ ), the radii of the two patches $\left(r_{\mathrm{p} 1}\right.$ and $\left.r_{\mathrm{p} 2}\right)$ and the offset $d y_{\mathrm{c}}$ of the protruding pin in the $y$-direction.

This structure was analyzed by means of an efficient and robust implementation of the mode-matching technique. The application of this method requires the knowledge of the modal distributions in all cross sections along the transmission channel having as input port the coaxial cable and as output port the aperture. From Fig. 4, it is evident that the configuration under investigation contains a combination of rectangular and coaxial waveguide sections. As for the latter variant, it is noted that the following cases occur:

- circular waveguides with concentric circular inner conductors and 
- rectangular waveguides with both concentric and eccentric circular inner conductors.

The modal representation on the coaxial segments was calculated by means of the BI-RME method [23] while that on the rectangular waveguide segments is known analytically. The transmission channel is completed by including a load that mimics the radiation into the free-space and (possibly) that accounts for the mutual coupling between the elements in the array configuration. The full-wave technique that is employed for calculating the load's GAM will be introduced at Sections III-C and D.

Let now $N_{\mathrm{c}}$ and $N_{\mathrm{a}}$ be the number of modes that are accounted for at the input (the coaxial cable) and output (aperture) ports of each of the 63 radiators pertaining to the shared aperture depicted in Fig. 2. The application of the mode-matching method finally yields a GAM for each radiator in the array, that interrelates the currents and the voltages at the above indicated ports by means of the relation

$$
\left[\begin{array}{c}
\boldsymbol{I}_{\mathrm{C}_{i}} \\
\boldsymbol{I}_{\mathrm{a}_{i}}
\end{array}\right]=\left[\begin{array}{ll}
\boldsymbol{Y}_{11_{i}} & \boldsymbol{Y}_{12_{i}} \\
\boldsymbol{Y}_{21_{i}} & \boldsymbol{Y}_{22_{i}}
\end{array}\right] \cdot\left[\begin{array}{l}
\boldsymbol{V}_{\mathrm{c}_{i}} \\
\boldsymbol{V}_{\mathrm{a}_{i}}
\end{array}\right], i=1, \ldots, 63
$$

where the index $i$ collectively identifies all the $\mathrm{R}_{\mathrm{H}}$ and $\mathrm{R}_{\mathrm{L}}$ radiators pertaining to the shared aperture; $\boldsymbol{I}_{\mathrm{C}_{i}}$ denote the column vector of equivalent modal currents at the coaxial port $i$ (its length being $N_{\mathrm{c}}$ ); $\boldsymbol{I}_{\mathrm{a}_{i}}$ denote the column vector of equivalent modal currents at the aperture port $i$ (its length being $N_{\mathrm{a}}$ ); $\boldsymbol{V}_{\mathrm{c}_{i}}$ denote the column vector of equivalent modal voltages at the coaxial port $i$ (its length being $N_{\mathrm{c}}$ ); $\boldsymbol{V}_{\mathrm{a}_{i}}$ denote the column vector of equivalent modal voltages at the aperture port $i$ (its length being $N_{\mathrm{a}}$ ); $\boldsymbol{Y}_{11_{i}}, \boldsymbol{Y}_{12_{i}}, \boldsymbol{Y}_{21_{i}}$ and $\boldsymbol{Y}_{22_{i}}$ are matrices of dimensions $N_{\mathrm{c}} \times N_{\mathrm{c}}, N_{\mathrm{c}} \times N_{\mathrm{a}}, N_{\mathrm{a}} \times N_{\mathrm{c}}$ and $N_{\mathrm{a}} \times N_{\mathrm{a}}$, respectively, containing the admittances coupling the relevant equivalent modal currents and voltages.

Note that, since the elements in the $\mathrm{SA}_{\mathrm{H}}$ and $\mathrm{SA}_{\mathrm{L}}$ subarrays were taken to be, respectively, identical, two sets of admittance matrices of the type indicated in (4) are sufficient for a complete description of the problem at hand.

In a first approximation, the transmission channel's load was taken to account for the radiation into the free-space of isolated elements, located on an infinitely extended flange. A parameter study, using as computational engine the numerical approach described earlier, has provided the following optimal sets of dimensions

- for the $\mathrm{R}_{\mathrm{H}}$ radiators: $a=b=12 \mathrm{~mm}, r_{\mathrm{p} 1}=5 \mathrm{~mm}$, $r_{\mathrm{p} 2}=4 \mathrm{~mm}$ and $d y_{\mathrm{c}}=4 \mathrm{~mm}$;

- for the $\mathrm{R}_{\mathrm{L}}$ radiators: $a=b=14 \mathrm{~mm}, r_{\mathrm{p} 1}=5.5 \mathrm{~mm}$, $r_{\mathrm{p} 2}=4.5 \mathrm{~mm}$ and $d y_{\mathrm{c}}=4.5 \mathrm{~mm}$.

Moreover, it was observed that accounting for the following sets of modes at the input and output ports suffices for ensuring the accuracy of the computed results

- $N_{\mathrm{c}}=7$ circular coaxial waveguide modes associated with the coaxial cable, i.e., the fundamental TEM-mode and $6 T E$-modes having the cut-off frequency below 100 $\mathrm{GHz}$ and

- $N_{\mathrm{a}}=10$ waveguide modes associated with the rectangular waveguide section that corresponds to the aperture [see Fig. 4(b)], i.e., $T E_{10}, T E_{01}, T E_{11}, T M_{11}, T E_{20}$, $T E_{02}, T E_{21}, T M_{21}, T E_{12}, T M_{12}$.

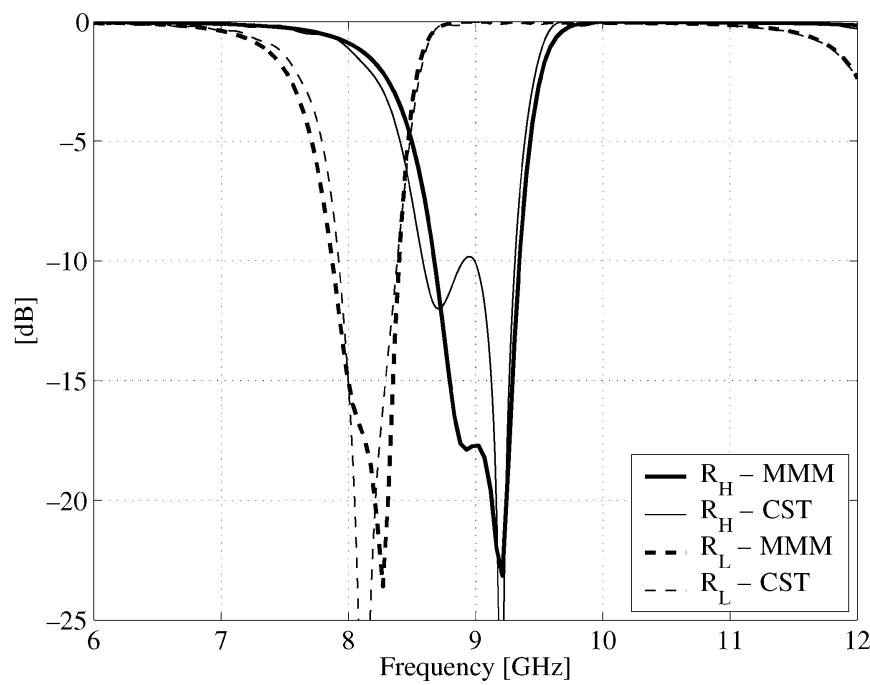

Fig. 5. Frequency dependence of the reflection coefficient in the case of isolated elements. Solid lines - the "type H" radiator; dashed lines - "type L" radiators. Thick lines: mode-matching analysis; thin lines: FDTD analysis.

With these choices, the frequency behavior of the reflection coefficient was examined for both types of radiators, the results being given in Fig. 5. From that behavior, it can be derived that two bands $7.9 \ldots 8.4[\mathrm{GHz}]$ and $8.6 \ldots 9.3[\mathrm{GHz}]$ (separated by a narrow gap of $0.2 \mathrm{GHz}$ ) are obtained for a voltage standing wave ratio VSWR $=2$. The two bands collectively yield a fractional bandwidth of $14 \%$, that exceeds the proposed design requirement $(10 \%)$.

The mode-matching results were also validated by means of a commercial software package (CST - Microwave Studio), the results being in good agreement. The small differences observed are a result of the fact that, while in the mode-matching model the metallic flange at the aperture was of infinite extent, the CST model employs a finite flange of dimensions $a=b=$ $50 \mathrm{~mm}$ [see Fig. 4(a)]. As regards the computation times required for solving the internal problem, it is mentioned that the mode-matching analysis requires approximately $3 \mathrm{~min}$ on a Pentium $4 \mathrm{CPU} / 2.4 \mathrm{GHz}$ equipped personal computer that has 256 MB of internal memory, while the CST code needed for the same task about 2 min on an identical machine.

\section{Evaluation of the Radiation Properties of Finite, Nonperiodic Arrays}

In Section III-A, the radiation properties were examined for the case of ideal, uncoupled radiators. However, in an interwoven, sparse array configuration, these assumptions fail to account appropriately for the electromagnetic phenomena associated with such complex structures. The performances of the individual radiators and of the array are strongly influenced by the concrete radiation environment (neighboring elements), an assessment that was substantiated in [27].

The theoretical background for the analysis of the radiation from rectangular apertures and for evaluating the mutual coupling between arbitrary apertures relies on the methodology presented in [21] and [22]. However, the straightforward application of that methodology in the case of large finite, sparse arrays (containing hundreds, possibly thousands of elements) is prohibitive from the point of view of the computing resources. 
For still ensuring the feasibility of the numerical calculations, the following observations turn out to be of key relevance.

- The GAM of an array antenna consisting of rectangular apertures includes partial GAM-s evaluated for doublets of apertures, only (the self-admittance being a particular case, in which an aperture is coupled with itself).

- For widely spaced apertures, the mutual coupling is small enough for being neglected in practical applications.

These observations allowed conceiving an effective computational strategy. It is hereafter exemplified for the case of an interwoven shared aperture antenna consisting of two differently sized apertures (the kind of which are investigated in this contribution).

1) Since two differently sized apertures are included in the array, three types of partial GAM-s need to be computed, only. They refer to the cases of couplings between identical elements (2 GAM-s) and of couplings between different elements (the third GAM). It is stressed that these partial GAM-s refer exclusively to doublets of apertures.

2) Due to the fact that above a certain interelement distance the mutual coupling can be neglected, it is sufficient to compute the partial GAM-s for doublets falling within a beforehand specified area of relatively small dimensions.

3) Assuming that the elements in the shared aperture antenna are deployed on a fixed grid and by taking into account the fact that the couplings are evaluated for an area of reduced dimensions, it can be concluded that the three types of partial GAM-s need to be computed for a limited number of positions, only. These results can be precooked for a given combination of aperture dimensions and grid steps and be subsequently employed in a "table look-up" manner for evaluating the properties of any array architecture that complies to the chosen design parameters (aperture dimensions and grid steps).

This computational strategy was implemented in a Matlab code, that has been run on a Pentium $4 \mathrm{CPU} / 2.4 \mathrm{GHz}$ equipped personal computer that has $256 \mathrm{MB}$ of internal memory. In the case of the shared aperture depicted in Fig. 2, the partial GAM-s were computed for $7 \times 7$ generic cells. The GAM-s were evaluated for 32 frequency points, in a range of $7, \ldots, 10 \mathrm{GHz}$. With these settings, the computational times for filling in the GAM-s were of $6 \mathrm{~h}$ in the case of the differently sized apertures and of 2 $\mathrm{h}$ and $40 \mathrm{~min}$ in the case of the couplings between identical apertures. Although these computation times may seem too large for the examined case, it is stressed that, by using the thus precalculated results, it is possible to perform the analysis of arrays of arbitrary size and architecture, as long as they use the same grid steps and aperture dimensions.

With respect to the details of the computer code implementation, it is noted that several mathematical artifices were employed for increasing the efficiency of the computations. For example, by partially solving the integrals occurring in the case of the evaluation of the self-admittances in an analytical manner, the relevant computation time amounted to only $47 \mathrm{~s}$. However, the bulk of the computation time was spent for estimating the couplings between different apertures. One option that could significantly increase the efficiency of these calculations is to employ the far-field approximation, as described in [28]. However, this approach fails in the case of closely packed apertures, that includes the vast majority of the situations encountered in practical antenna arrays. Another option is adopting the reduced order polynomial approximation that was originally introduced in [29] and demonstrated for the case of multimodal analyzes in [30].

Upon applying this computational strategy, the set of all equivalent modal currents and voltages at the 63 apertures in Fig. 2 can now be related as

$$
-\left[\begin{array}{c}
\boldsymbol{I}_{\mathrm{a}_{1}} \\
\boldsymbol{I}_{\mathrm{a}_{2}} \\
\vdots \\
\boldsymbol{I}_{\mathrm{a}_{63}}
\end{array}\right]=\mathrm{GAM}^{\text {ext }} \cdot\left[\begin{array}{c}
\boldsymbol{V}_{\mathrm{a}_{1}} \\
\boldsymbol{V}_{\mathrm{a}_{2}} \\
\vdots \\
\boldsymbol{V}_{\mathrm{a}_{63}}
\end{array}\right]
$$

in which $\mathrm{GAM}^{\text {ext }}$ represents the $630 \times 630$ matrix of coupling admittances. The minus sign in (5) accounts for the fact that the modal currents at the aperture are taken to be directed toward the generator. Note that the fact that the partial GAM-s were evaluated for $7 \times 7$ generic cells implies that some of the entries in $\mathrm{GAM}^{\text {ext }}$ are intrinsically 0 (for example, the coupling between the modal quantities pertaining to the elements $1_{\mathrm{L}}$ and $6_{\mathrm{L}}$ ).

\section{The Aggregate Problem}

The final step in the numerical analysis is to integrate the internal and the external problems. Initially, the equations pertaining to the internal problem (4), that were written for each element, separately, are now assembled in a single matrix equation that is valid for the complete array. Furthermore, by combining this system of equations with the equations given in (5), one can easily find an input impedance matrix that relates the modal equivalent currents and voltages at the input port of each elementary radiator in the array configuration.

In the demonstrated example, the input impedance matrix is associated with the coaxial cables (the true input ports of the examined configuration) and incorporates the effect of the radiation into the free-space and of the mutual couplings, as well. This matrix can be, in turn, employed for computing the generalized scattering matrix of the complete structure and, hence, of the reflection coefficients pertaining to the modes at the input port.

The frequency behavior of the reflection coefficients referring to the fundamental TEM-mode on the coaxial cables are depicted in Fig. 6(a) and (b) for the two subarrays, separately. These plots clearly illustrate the fact that the matching properties of the elementary radiators are affected significantly when they are placed in an array environment. The variation in the matching properties may result in the deterioration of the antenna efficiency (in the case when parts of the operational spectrum are not radiated by the elements) and, at the same time, introduces an amplitude modulation that has consequences in the radiation pattern of the shared aperture antenna. In the concrete case of the currently examined antenna, the elements $8_{\mathrm{H}}$, $14_{\mathrm{H}}, 18_{\mathrm{H}} 6_{\mathrm{L}}, 9_{\mathrm{L}}$, and $24_{\mathrm{L}}$ (see Fig. 2 for their location) have a visibly narrower bandwidth while $12_{\mathrm{H}}, 16_{\mathrm{H}}, 26_{\mathrm{L}}$, and $27_{\mathrm{L}}$ have a slightly enlarged bandwidth. However, no correlation between the location in the array and the manner in which the bandwidth is affected can be derived from comparing Figs. 2 and 6. 


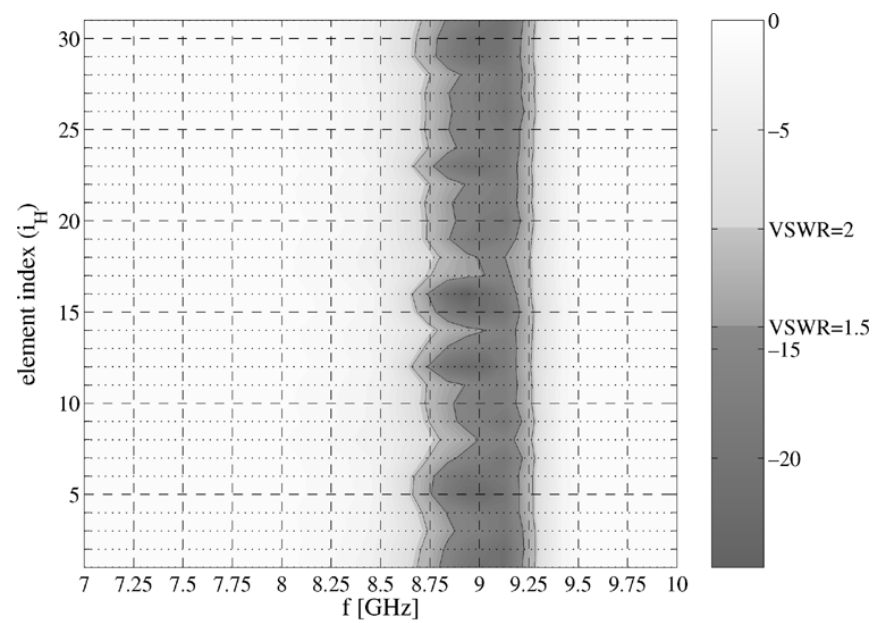

(a)

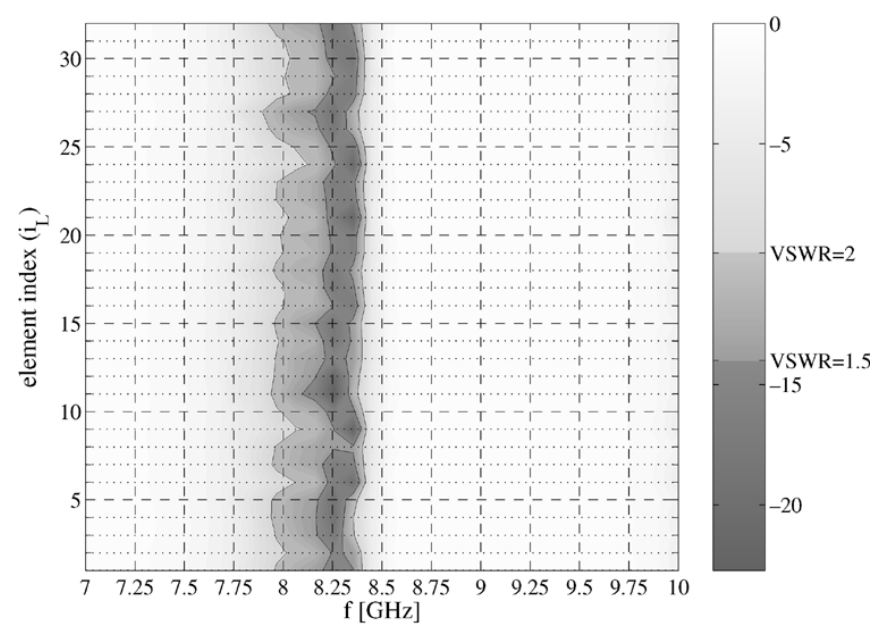

(b)

Fig. 6. Frequency dependence of the active reflection coefficient for each elementary radiator in the two subarrays; the solid contours indicate the levels corresponding to VSWR $=2$ and VSWR $=1.5$, respectively. (a) Subarray $\mathrm{SA}_{\mathrm{H}}$. (b) Subarray $\mathrm{SA}_{\mathrm{L}}$.

At this point of the design strategy, the behavior of the embedded elements can be judged. In the case when the matching properties of the elementary antennas are considered satisfactory, the elements in each subarray are kept identical. However, when the shared aperture contains elements that do not meet the bandwidth requirements, supplementary measures need to be taken. To this end, the design tool discussed in this paper includes an automatic procedure for tuning the elementary antennas individually. The method is based on a mathematical programming algorithm (conjugate-gradient) that searches for the optimum antenna dimensions (coaxial cable offset and radius of the stacked patches) that minimize the input reflection coefficient over a certain frequency range. For evaluating the input admittance at each feeding point, the radiation from the aperture is replaced by an equivalent impedance, which takes into account the coupling effect and has different values for each antenna in the shared aperture. Hence, the external problem need not being solved again. Moreover, due to the modularity of the mode-matching method, there are but a few blocks of the internal problem that need to be recalculated. Apart from its intrinsic computational benefits, the tuning methodology based on

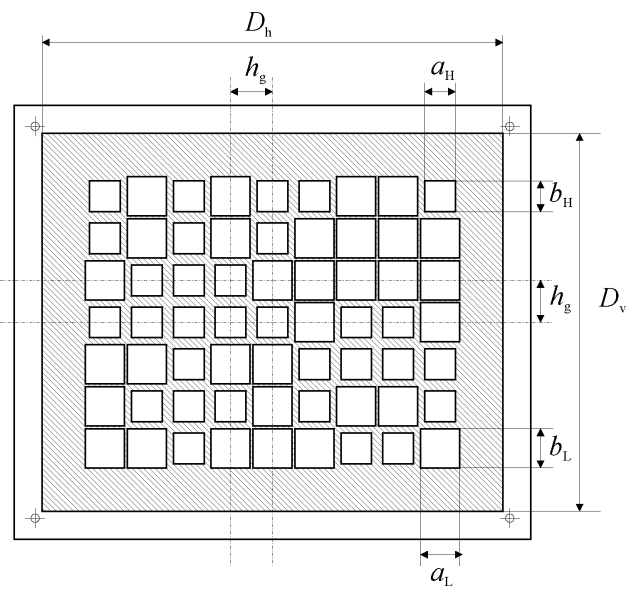

Fig. 7. Fully assembled shared aperture (top view). Principal dimensions: $D_{\mathrm{h}}=165 \mathrm{~mm}, D_{\mathrm{v}}=135 \mathrm{~mm}, a_{\mathrm{H}}=12 \mathrm{~mm}, b_{\mathrm{H}}=12 \mathrm{~mm}, a_{\mathrm{L}}=14 \mathrm{~mm}$, $b_{\mathrm{L}}=14 \mathrm{~mm}$, and $h_{\mathrm{g}}=15 \mathrm{~mm}$.

the changing of the offset of the feeding point and of the radius of the two patches allows for the shifting of the bandwidth of the elementary radiator over a large frequency range.

In the particular configuration under investigation, it is indicated that the results depicted in Fig. 6 refer to the case of subarrays consisting of identical elements. Since the matching properties of the resulting system were appreciated as being satisfactory, no additional individual tuning phase was carried out.

The layout of the completely assembled shared aperture antenna is presented in Fig. 7. The aggregate thickness of the ensemble amounts to $2.3 \mathrm{~mm}$ (the SMA connectors excluded), that is consistent with the originally assumed design requirement for obtaining a low-profile product. Note that Fig. 7 can also be interpreted as the mask for etching the upper layer of the antenna, when implemented in a printed, stratified technology.

\section{CONCLUSION AND Discussion}

A consistent strategy for the design of finite array antennas consisting of differently sized radiating elements was discussed. By resorting to a shared aperture array architecture, an increased bandwidth that is instrumental for implementing multifunctionality of the antenna systems was obtained. An effective computational formalism, incorporating a full-wave analysis of the individual radiators in conjunction with a full-wave investigation of the phenomena related to the radiation from finite, nonperiodic arrays, was described. The formalism allows for an accurate analysis of the complete transmission channel, starting from the feeding coaxial cable, that is commonly the true input port of antennas. At the same time, the advocated numerical strategy enables a full-wave analysis of large, nonperiodic array antennas containing differently sized elements, while maintaining computational feasibility.

The design strategy lends itself to several extensions. First, it is observed that, once the external problem was addressed and the $\mathrm{GAM}^{\text {ext }}$ matrix was stored, the individual radiators can be designed without requiring the (highly time consuming) evaluation of the radiation part. Furthermore, the complete analysis of elementary antennas is computationally very efficient. Corroborating these two features, it becomes realistic to devise an auto- 
matic tuning procedure, using the geometrical parameters of the internal problem as variables subject to optimization. Second, the "table look-up" procedure that was employed for solving the external problem opens the possibilities to examine array antennas having a large number of elements in configurations combining elements of various sizes. In a multifunctional array, this provides the possibility to a priori estimate the interaction between the systems sharing the antenna resources.

\section{REFERENCES}

[1] N. Fourikis, Phased Array-Based Systems and Applications. New York: Wiley, 1996.

[2] T. A. Axness, R. V. Coffman, B. A. Kopp, and K. W. O'Haver, "Shared aperture technology development," in The John Hopkins APL Tech. Dig., vol. 17, Jul.-Sep. 1996, pp. 285-294.

[3] C. Hemmi, R. T. Dover, F. German, and A. Vespa, "Multifunction wideband array design," IEEE Trans. Antennas Propag., vol. 47, pp. 425-431, Mar. 1999.

[4] D. G. Shively and W. L. Stutzman, "Wideband arrays with variable element sizes," Proc. Inst. Elect. Eng. Microwaves, Antennas Propagation, vol. 137, pp. 238-240, Aug. 1990.

[5] D. M. Pozar and S. D. Targonski, "A shared-aperture dual-band dualpolarized microstrip array," IEEE Trans. Antennas Propag., vol. 49, pp. 150-157, Feb. 2001.

[6] H. Loui, J. P. Weem, and Z. Popovic, "A dual-band dual-polarized nested Vivaldi slot array with multilevel ground plane," IEEE Trans. Antennas Propag., vol. 51, pp. 2168-2175, Sep. 2003.

[7] I. E. Lager, C. I. Coman, and L. P. Ligthart, "The shared aperture, sparse array antenna approach to designing broadband array antennas," in Proc. MSWM '04 - 5th Int. Kharkov Symp. Physics and Engineering of Microwaves, Millimeter and SubMillimeter Waves, 2004, pp. 91-96.

[8] J.-C. Cheng, N. I. Dib, and L. P. B. Katehi, "Theoretical modeling of cavity-backed patch antennas using a hybrid technique," IEEE Trans. Antennas Propag., vol. 43, pp. 1003-1008, Sep. 1995.

[9] A. K. Bhattacharyya, "A modular approach for probe-fed and capacitively coupled multilayered patch arrays," IEEE Trans. Antennas Propag., vol. 45, pp. 193-202, Feb. 1997.

[10] S. D. Targonski, R. B. Waterhouse, and D. M. Pozar, "Design of wide-band aperture-stacked patch microstrip antennas," IEEE Trans. Antennas Propag., vol. 46, pp. 1245-1251, Sep. 1998.

[11] R. Garg, P. Bhartia, I. Bahl, and A. Ittipiboon, Microstrip Antenna Design Handbook. Boston, MA: Artech House, 2001.

[12] M. A. Khayat, J. T. Williams, D. R. Jackson, and S. A. Long, "Mutual coupling between reduced surface-wave microstrip antennas," IEEE Trans. Antennas Propag., vol. 48, pp. 1581-1593, Oct. 2000.

[13] J.-M. Jin and J. L. Volakis, "A hybrid finite element method for scattering and radiation by microstrip path antennas and arrays residing in a cavity," IEEE Trans. Antennas Propag., vol. 39, pp. 1598-1604, Nov. 1991.

[14] M. A. González de Aza, J. A. Encinar, and J. Zapata, "Radiation pattern computation of cavity-backed and probe-fed stacked microstrip patch arrays," IEEE Trans. Antennas Propag., vol. 48, pp. 502-509, Apr. 2000.

[15] M. A. González de Aza, J. Zapata, and J. A. Encinar, "Broad-band cavity-backed and capacitively probe-fed microstrip patch arrays," IEEE Trans. Antennas Propag., vol. 48, pp. 784-789, May 2000.

[16] C. I. Coman, I. E. Lager, and L. P. Ligthart, "Sparse array antennas," in Proc. Workshop EuMC WS 5. Multifunction Radar Front End Design and Management, 2003, pp. 19-22.

[17] L. E. Kopilovich and L. G. Sodin, "Two-dimensional aperiodic antenna arrays with a low side-lobe level," Proc. Inst. Elect. Eng. Microwaves, Antennas and Propagation, vol. 138, pp. 233-237, Jun. 1991.

[18] D. G. Leeper, "Isophoric arrays - Massively thinned phased arrays with well-controlled sidelobes," IEEE Trans. Antennas Propag., vol. 47, pp. 1825-1835, Dec. 1999.

[19] C. I. Coman, I. E. Lager, and L. P. Ligthart, "Shared aperture sparse array antenna for wide band applications," in Proc. European Microwave Week 2003, 2003, pp. 515-518.

[20] T. Weiland, "Time domain electromagnetic field computation with finite difference methods," Int. J. Numerical Modeling: Electronic Networks, Devices and Fields, vol. 9, pp. 295-319, Jul. 1996.

[21] T. S. Bird, "Analysis of mutual coupling in finite arrays of different-sized rectangular waveguides," IEEE Trans. Antennas Propag., vol. 38, pp. 166-172, Feb. 1990.

[22] T. S. Bird and D. G. Bateman, "Mutual coupling between rotated horns in a ground plane," IEEE Trans. Antennas Propag., vol. 42, pp. 1000-1006, Jul. 1994.
[23] G. Conciauro, M. Gugliemi, and R. Sorrentino, Advanced Modal Analysis. CAD Techniques for Waveguide Components and Techniques. Chichester, U.K.: Wiley, 1999.

[24] L.-G. Huss, A. Pohl, R. Gunnarsson, A. Ouacha, and A. Gustafsson, "Array antenna module for an X-band smart skin radar receiver," in Proc. Antenn03, Nordic Antenna Symp., 2003, pp. 273-278.

[25] A. Gustafsson, R. Malmqvist, L. Pettersson, L.-G. Huss, M. Alfredson, S. Lindström, I. Ferrer, P. Grahn, S. Leijon, C. Samuelsson, T. Nilsson, A. Pohl, A. Ouacha, B. Carlegrim, S. Hagelin, and R. Erickson, "A very thin and compact smart skin X-band digital beamforming antenna," in Proc. 1st European Radar Conf., 2004, pp. 313-316.

[26] High frequency laminates literature index. Rogers CorporationAdvanced Circuit Materials Division. [Online]. Available: http://www. rogerscorporation.com

[27] M. G. Bray, D. H. Werner, D. W. Boeringer, and D. W. Machuga, "Optimization of thinned aperiodic linear phased arrays using genetic algorithms to reduce grating lobes during scanning," IEEE Trans. Antennas Propag., vol. 50, pp. 1732-1742, Dec. 2002.

[28] F. J. S. Moreira and J. R. Bergmann, "Asymptotic approximations for the mutual coupling in finite arrays of rectangular waveguides," in Proc. 8th Int. Conf. Antennas and Propagation, vol. 2, 1993, pp. 776-779.

[29] M. C. Bailey, "Technique for extension of small antenna array mutualcoupling data to larger antenna arrays," NASA Langley Res. Center, Hampton, VA, NASA Tech. Paper 3603, 1996.

[30] C. I. Coman, I. E. Lager, and L. P. Ligthart, "Optimization of linear sparse array antennas consisting of electromagnetically coupled apertures," in Proc. 1st Eur. Radar Conf., 2004, pp. 301-304.

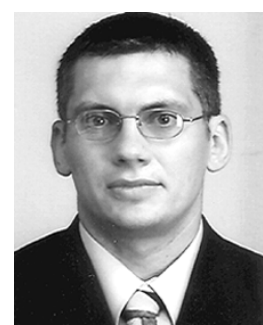

Cristian I. Coman (S'04) was born in Tirnăveni, Romania, on March 14, 1972. He received the Engineer's degree from the Military Technical Academy, Bucharest, in 1996.

$\mathrm{He}$ is currently pursuing the Ph.D. degree in electrical engineering at the Delft University of Technology, Delft, The Netherlands. His current interests include sparse array antennas, shared aperture antennas, computational electromagnetics and radar signal processing.

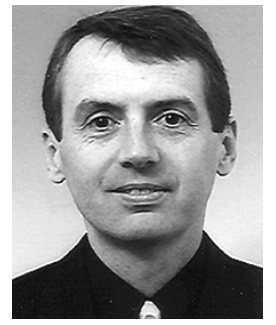

Ioan E. Lager (M'01) was born in Braşov, Romania, on September 26, 1962. He received the Engineer's degree from the Transilvania University, Brasov, in 1987 and Doctor's degrees from the Delft University of Technology, Delft, the Netherlands, and from the Transilvania University, in 1996 and 1998, respectively.

Since 1997, he has been with the Delft University of Technology, first with the Laboratory of Electromagnetic Research and, since 1998, with the International Research Centre for TelecommunicationsTransmission and Radar (IRCTR), where he coordinates the applied electromagnetics-related activities. He authored one monograph and more than 25 refereed publications. His principal scientific interests concern the (technological) design of antennas and the field of computational electromagnetics.

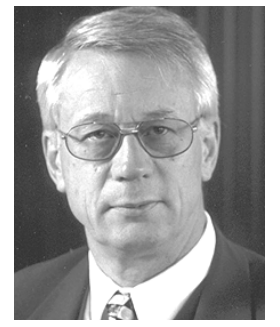

Leo P. Ligthart (M'94-SM'95-F'02) was born in Rotterdam, The Netherlands, on September 15, 1946. $\mathrm{He}$ received an Engineer's degree (cum laude) and a Doctor of Technology degree from Delft University of Technology, Delft, The Netherlands, in 1969 and 1985, respectively. He received Doctorates (honoris causa) from Moscow State Technical University of Civil Aviation in 1999 and Tomsk State University of Control Systems and Radioelectronics in 2001.

Since 1992, he has held the Chair of Microwave Transmission, Radar and Remote Sensing in the Department of Information Technology and Systems, Delft University of Technology. In 1994, he became Director of the International Research Center for Telecommunications- Transmission and Radar. His principal areas of specialization include antennas and propagation, radar and remote sensing, but he has also been active in satellite, mobile, and radio communications. 